\title{
V14 - SACCHAROMYCES BOULARDII AS AN ALTERNATIVE EXPRESSION SYSTEM FOR MOLECULES WITH BIOTHERAPEUTICAL APPLICATIONS
}

Bruno Douradinha $^{1,2}$, Vivian Castelo Branco Reis ${ }^{3}$, Fernando Araripe Gonçalves Torres ${ }^{3}$, Jared David Evans ${ }^{2}$, Ernesto Torres Azevedo Marques Jr²

1. Fondazione Rimed, Palermo, Italy, 2. University of Pittsburgh Center for Vaccine Research, Pittsburgh (PA), USA, 3. Centro de Biotecnologia Molecular, Universidade de Brasília, Brasília, Brazil

Objectives: This work shows that Saccharomyces boulardii (S. boulardii), a probiotic yeast related to Saccharomyces cerevisiae (S. cerevisiae) shares with the latter several genetic features which prompt its use as an alternative expression system for molecules with potential biotherapeutical applications.

Methodologies: $S$. boulardii is a probiotic yeast related to Saccharomyces cerevisiae ( $S$. cerevisiae), but with distinct genetic, taxonomic and metabolic properties. S. cerevisiae has been extensively used in biotechnological applications. Currently, many strains are available and multiple genetic tools have been developed, which allow the expression of several exogenous proteins of interest with applications in the fields of medicine, biofuels, food industry and scientific research, among others. Although S. boulardii has been widely studied due to its probiotic properties against several gastrointestinal tract disorders, very few studies addressed the use of this yeast as a vector for expression of foreign genes of interest with biotechnological applications. Here we applied to the probiotic yeast a simple plasmid transformation technique based on the lithium acetate protocol used for S. cerevisiae with minor modifications. We also tried different protocols for screening based on the protoplast formation. We further screened by PCR the genome of $S$. boulardii for the presence of promoters and other molecules of interest (PGK1, PYK1, ENO1, ADH1, REP1, REP2, CAN1, TAF10, AGA1 and $\delta$ transposons).

Results: We developed a simple protocol to efficiently perform plasmid DNA transformation into $S$. boulardii and consequent screening of successful transformants. We also show that several genes frequently used in genetic manipulation of S. cerevisiae (e.g., promoters, resistance markers) are present in S. boulardii, but some of them are not eligible to be targeted during transformation of the latter. 
Conclusion: This work shows that although S. boulardii and S. cerevisiae are highly similar at several levels, not all tools used for genetic transformation of the latter can be applied to the probiotic yeast. This work has important applications toward the potential of $S$. boulardii as an expression system for genes of interest. 